\title{
Linking large and small computers
}

\author{
EDWARD GRUPSMITH \\ New York University, New York, New York 10003
}

\begin{abstract}
A PDP-8/I was connected to an IBM/370 via telephone. Programs enable the PDP-8 to send data to practically any large-scale computer having timesharing capabilities. These programs can be used as models by others wishing to analyze data on a large computer.
\end{abstract}

Many people who use minicomputers to run experiments have a problem when it comes to data reduction and analysis. Minicomputers are generally not equipped to do some types of computing efficiently. Small on-line computers typically have limited amounts of core and are rarely configured with plotters or line printers. On-line storage of data is also usually very limited. Truly comprehensive software statistical packages do not exist for minicomputers. On the other hand, large-scale computers are designed for data reduction. They generally have lots of on-line storage capacity as well as plotters and line printers. In addition, software packages such as SPSS, BMD, and DATATEXT are usually available and, through their use, the time needed for data analysis can be drastically reduced.

How can information be transferred from a small to a large computer? A card punch or industrial compatible magnetic tape can be interfaced to the minicomputer, but magnetic tape and card punch equipment are expensive, both to purchase and to maintain. An alternative is to attach an acoustic coupler to a terminal, assuming the large-scale computer has a timesharing system. Then you can type in data by hand. Since your data is already on a medium that is readable by the small computer, the next step is to make the larger "host" computer "think" that the mini is a terminal. Then data can be sent over telephone lines into the larger computer's timesharing system.

\section{DATA TRANSMISSION PROCEDURE}

In the New York University psychology department, a Digital Equipment Corporation PDP-8/I is used to run human memory and learning experiments. The PDP-8/I system is equipped with $16 \mathrm{~K}$ of core, a standard ASR-33 Teletype, DECtapes, and a high-speed paper-tape reader and punch. Display equipment has been added in order to run experiments (see Aaronson \& Brauth, 1972; Aaronson, Grupsmith, \& Ohman, 1975).

The preparation of this paper was supported in part by Grant MH16496, awarded to Doris Aaronson from the National Institute of Mental Health. I would like to thank Doris Aaronson for helpful comments on an earlier draft. Requests for reprints should be sent to Edward Grupsmith, Department of Psychology, New York University, 4 Washington Place, Room 873, New York, New York 10003.
Figure 1 shows the hardware configuration used to transfer data. To equip the PDP as an "intelligent terminal" (Vaughan, Note 1), a DEC BD50, which is an RS232 compatible serial interface, and an Omnitec Model 701R telephone coupler were added to the system. The large computer is an IBM $370 / 145$ equipped with an IBM 3705 communications controller. Two timesharing systems are available on the IBM, Wylbur and CMS. Both have facilities for inputting and storing data files.

\section{Programs}

The PDP-8/I program required to converse with the "host" computer had to meet certain specifications. The first consideration was transmission rate. We were tying into a system meant to interact with terminals. The 3705 communications controller on the IBM accepts input on dial-up lines at 110,150 , and 300 baud (10, 15 , and 30 characters/sec). The clock on the BD50 was set at 300 baud.

A second consideration was code format. The PDP-8/I uses 8-bit ASCII codes to transmit and store data. The 3705 accepts this code as well as 6- and 7-bit ASCII and various IBM codes. Therefore, no code translation was needed.

The third consideration was the program used by the IBM to receive our PDP data. It was decided that the PDP should interact with the editor in either CMS or Wylbur. Most computer editors operate in two modes.

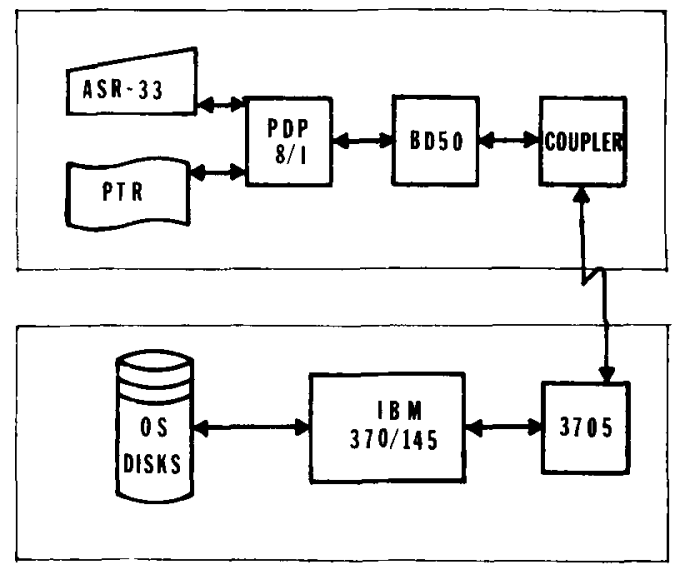

Figure 1. Hardware used for data link. 
In edit mode commands are issued to manipulate and change text that is already stored in the editor. The other mode is input mode, in which text is generally entered on-line, one line at a time. All editors are initially in edit mode and a command can be issued which places them into input mode. After the desired input is typed, a return to edit mode can be made by typing a carriage return twice in CMS or by hitting the break key for Wylbur. Common to both input and edit modes is that a prompt must be received from the IBM before a new line of information can be typed by the user.

While the IBM editor is in edit mode, we can interact with it using the PDP's Teletype. We then shift to input mode and read data from the PDP paper tapes. These data are sent to the IBM with no further intervention from the user. Thus, the PDP program must be able to recognize a normal prompt from the IBM for more information, in which case another line is sent. The PDP program has to discriminate a normal from an error prompt, such as a "read" error or any other type. When the IBM signals an error condition, the PDP program returns control to the user. The user then takes corrective action.

The PDP-8 program is designed to operate in two modes: conversational or paper tape. In conversational mode anything typed on the PDP Teletype is sent to the IBM, and anything received from the IBM is printed on the Teletype. When in conversational mode, two special characters are recognized by the PDP program. A back arrow (octal code 337 ) puts the program into paper-tape mode, and an up arrow (octal 336) retransmits the last line read from the paper tape.

In paper-tape mode a line of input is read from the tape reader and transmitted over the phone line to the IBM. Any prompt or response from the IBM is received by the PDP and stored in a software buffer. If it is a normal prompt, the next line is read and transmitted. If it is an error prompt, the message received is printed on the PDP Teletype and control is returned to the user. All prompts end with an XON character (221 octal). All normal prompts are less than 14 characters long; all error prompts are greater than 14 characters.

In turning our computer into an "intelligent terminal," the decision was made to write the program as a fully interruptable system. That is, any device can demand the attention of the PDP computer. It does this by raising a flag. If the PDP's interrupt facility is enabled, the program being executed is stopped and control is transferred to a program located at Memory Location 1. Meanwhile, the next location to be executed from the interrupted program is stored at Location 0 . Interrupts are generated when a Teletype key is pressed or when the Teletype finishes printing a character. Similarly, the BD50 will generate an interrupt when a character is received or when it has finished transmitting a character. The paper-tape reader generates interrupts in a similar fashion.

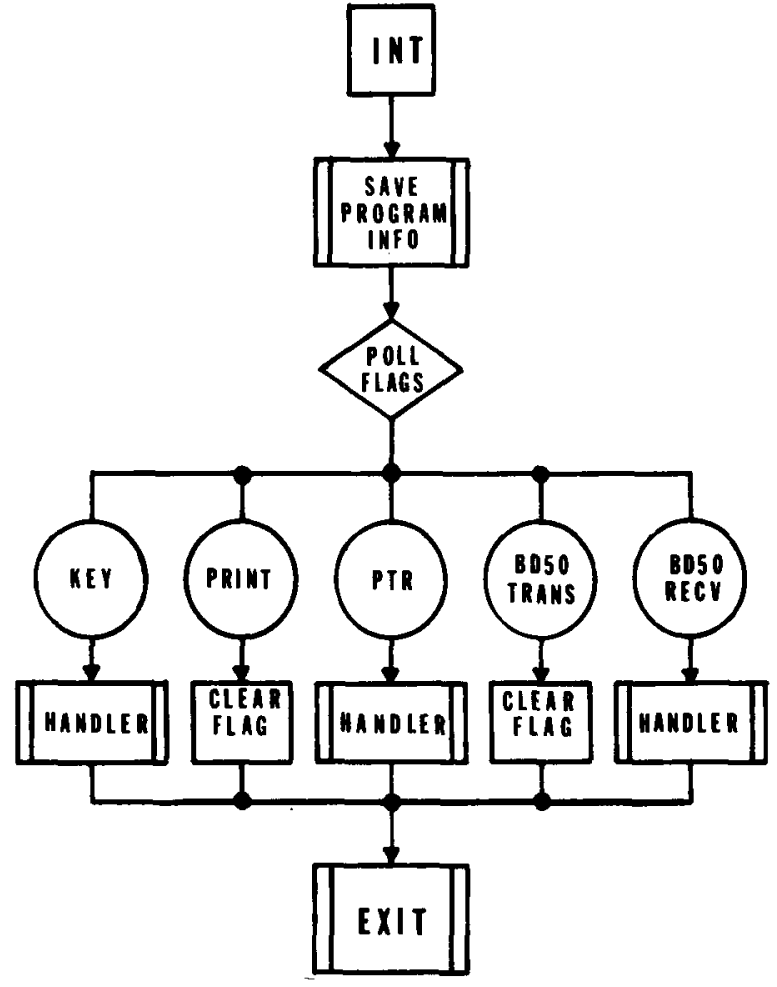

Figure 2. Interrupt program flowchart.

Figure 2 shows the flow of the interrupt-handling routine. When a device generates an interrupt, this program takes over. First, program information is saved, such as the contents of major registers. This information is later used to return to the main program from the interrupt-handling routines. Next, the flags of the devices attached to the computer are polled to determine which one generated the interrupt. Then a device-dependent subroutine is called that takes action appropriate to that device. If the printer generates an interrupt signaling that it had finished printing a character, then that flag is cleared, as well as a software flag that tells another part of the program that it is okay to print another character. If the Teletype keyboard causes the interrupt, then the character typed is stored in a software buffer. It then determines if the character is special, such as the back arrow, which puts the program into paper-tape mode. If the character is a back arrow, a subroutine is called that changes several software flags, indicating to the program that it is now in paper-tape mode. If the character typed is a normal one, a flag signifying that a character is waiting to be typed is set, and then an exit routine is executed.

Figure 3 shows the handler for the BD50 receiver connected to the PDP. This handler deals with characters received from the IBM. First, the character is read and the flag associated with the receiver is cleared. The character is then stored in a buffer. Next, determination is made as to which mode the data transfer program is in. If it is in conversational mode, a software flag, TFLAG, indicating that there is something to be typed, 


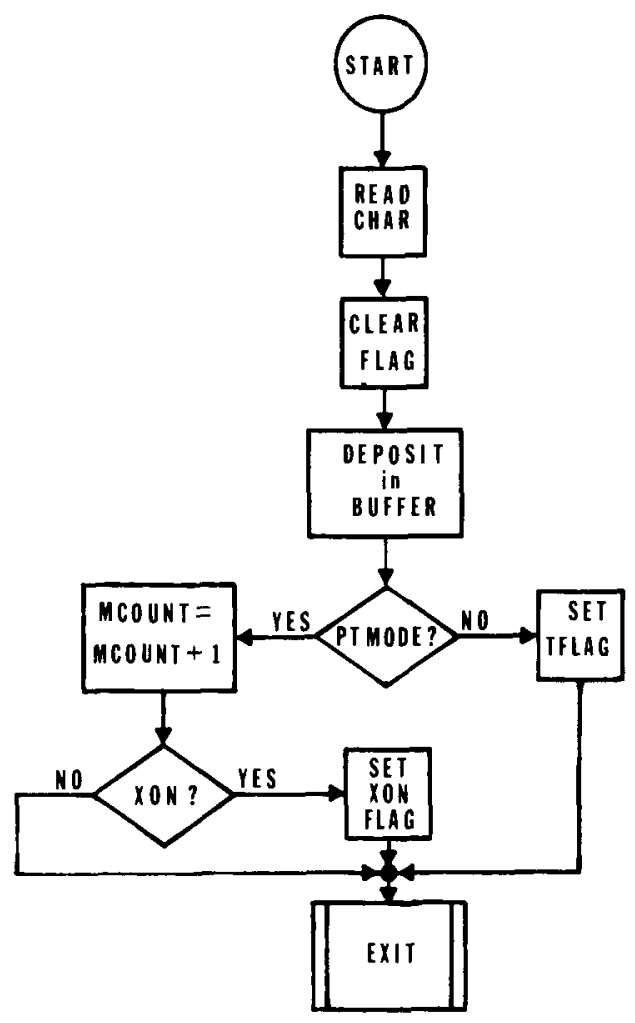

Figure 3. Flowchart for BD50 receiver handler.

is set and the program exits from the interrupt. If the program is in paper-tape mode, a counter, MCOUNT, is incremented to count the number of characters in the message. This information is later used to determine whether a normal prompt has been received (i.e., 14 characters or less). Next we determine if the character received is an XON, indicating the end of a prompt. If it is not an XON, we exit; if it is an XON, a software flag is set that indicates an XON has been received.

The main part of the program is a loop that interrogates the software flags set by the interrupt routines.

Figure 4 shows the conversational mode part of the program. To start, the mode must be ascertained. If in paper-tape PTMODE, we jump to another part of the program. If in conversational mode, we go into a loop which checks what must be done. First the sending flag, SSW, is checked to see if we have completed sending the last character. If not, we check other flags. If the transmission has been completed, we see if there is anything waiting to be sent (SFLAG). If SFLAG is set, a character is retrieved from the PDP buffer and sent to the IBM. Then the SSW flag is set, indicating that a character is being transmitted. After the transmission, an analogous loop handles typing onto the PDP Teletype. Steps in that loop are as follows: Are we in the act of typing? Is the typing flag set (TSW)? If not, is there anything to type? Is TFLAG set? If so, obtain the character and type it. Then set TSW, indicating that we are in the act of typing. When finished, go back and check on sending. The back and forth between sending and typing will continue until the user types a special character that piaces him into paper-tape mode.

Figure 5 shows the flow of the paper-tape mode



Figure 4. Flowchart for conversational mode.

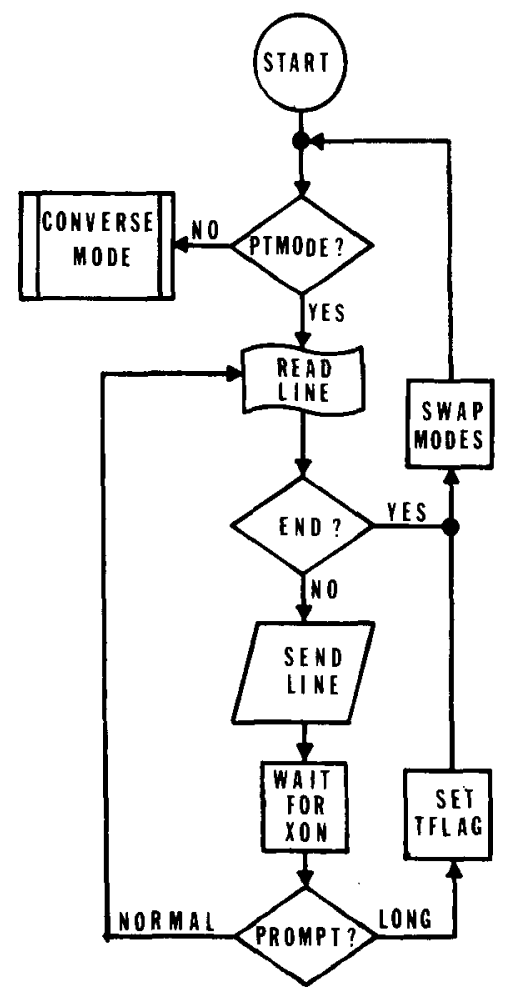

Figure 5. Flowchart for paper-tape mode. 
routine. Once in paper-tape mode (PTMODE), a line of text is read from the paper-tape reader and stored in a PDP buffer. If there is no tape in the reader, we return to conversational mode. If a good line, it goes to the IBM and waits for an XON character to be received. When XON is received, look at the length of the prompt received before the XON. If the prompt is of normal length, go back and read another line; if it is long, indicating an error message, a software flag is set, indicating that the last line received should be typed. Then we are returned to conversational mode.

This program has successfully transmitted many thousands of lines of data. Given the general flow of the program as described, similar programs can be written for practically any combination of mini and large-scale computers.

\section{REFERENCE NOTE}

1. Vaughan, J. The mini and micro as intelligent terminals. Paper presented at the Seventh Annual Meeting of the National Conference on the Use of On-Line Computers in Psychology, November 9, 1977.

\section{REFERENCES}

Aaronson, D., \& Brauth, S. SIMPLE guidelines for developing a computer-based laboratory. Behavior Research Methods \& Instrumentation, 1972, 4, 257-264.

Aaronson, D., Grupsmith, E., \& Ohman, D. SIMPLE control and measurement of auditory events. Behavior Research Methods \& Instrumentation, 1975, 7, 125-130. 